\title{
How to make sense of the jet correlations results at RHIC?
}

\author{
Jiangyong Jia ${ }^{\mathrm{a}}$ \\ Chemistry Department, Stony Brook University, Stony Brook, NY 11794, USA \\ Physics Department, Brookhaven National Laboratory, Upton, NY 11796, USA
}

Received: 30 September 2008 / Published online: 12 February 2009

(C) Springer-Verlag / Società Italiana di Fisica 2009

\begin{abstract}
We review the di-hadron correlation results from RHIC. A consistent physical picture was constructed based on the correlation landscape in $p_{T}, \Delta \phi, \Delta \eta$ and particle species. We show that the data are consistent with competition between fragmentation of survived jets and response of the medium to quenched jets. At intermediate $p_{T}$ where the medium response are important, a large fraction of trigger hadrons do not come from jet fragmentation. We argue that these hadrons can strongly influence the interpretation of the low $p_{T}$ correlation data. We demonstrate this point through a simple geometrical jet absorption model simulation. The model shows that the correlation between medium response hadrons dominates the pair yield and mimics the double hump structure of the away-side $\Delta \phi$ distribution at low $p_{T}$. This correlation was also shown to lead to complications in interpreting the results on reaction plane dependence and three particle correlations. Finally, we briefly discuss several related experimental issues which are important for proper interpretations of the experimental data.
\end{abstract}

PACS 25.75.-q

\section{Introduction}

Single jets and back-to-back di-jet pairs are important tools for studying the properties of the dense matter created in relativistic heavy-ion collisions. Due to difficulties of full jet reconstruction at RHIC, they are accessed through leading hadron spectra and di-hadron correlation. The primary handle for spectra analysis is the hadron $p_{T}$, whereas multiple handles can be used in di-hadron correlation analysis: the momentum of trigger $\left(p_{T}^{A}\right)$, momentum of partners $\left(p_{T}^{B}\right)$, $\Delta \phi$ and $\Delta \eta$. The possibility of varying all these variables leads to large amount of experimental data.

\footnotetext{
a e-mail: jjia@bnl.gov
}

A schematic illustration of the di-hadron correlation signal for unmodified jets (such as in $p+p$ collisions) is presented in left panel of Fig. 1.1. The signal appears as a narrow peak at $(\Delta \phi, \Delta \eta) \sim(0,0)$ and a broad peak at $\Delta \phi \sim \pi$ which is flat in $\Delta \eta$ up to $|\Delta \eta|<2$. The former corresponds to pairs from the same jet, while the latter corresponds to pairs from the away-side jet. The elongation of the awayside pairs in $\Delta \eta$ is due to longitudinal momentum imbalance between the two original partons that undergo hard-scatter process.

In $\mathrm{Au}+\mathrm{Au}$ collisions, jets are modified by the dense medium. In addition to the jet fragmentation component coming mainly from those jets that do not interact with the medium (due to surface emission or punch-through) [1,2], extensive experimental studies [3-6] have revealed additional medium response components as illustrated in the right panel of Fig. 1.1. These medium response components appear as three distributions that peaks at different $\Delta \phi$ locations: $\Delta \phi \sim 0, \pi \pm 1.1$, but are flat in $\Delta \eta$. The current focus of the field is to understand the interplay of the contributions from jet fragmentation and medium response.

Beyond this qualitative picture of the jet-medium interaction, the progress so far is limited in our understanding of the fundamental mechanisms in terms of the plasma properties. We do not yet fully understand the jet quenching, let alone the medium response mechanism. In a sense there are too much experimental information: new measurements are continuously being made, but the progress of integrating them into fundamental theoretical framework has been slow.

The reason for current impasse is two fold. Experimentally, there are assumptions made in the extraction of jet signal, such as the two-source model and ZYAM assumptions in background subtraction [3, 4]. There are also questions on how to deal with non-flow effects and event-by-event fluctuation in background subtraction. On the theory side, the medium response is intrinsically non-perturbative and difficult to model. Most calculations assume that there is a scale that separates jet energy loss and medium response $[7,8]$. 


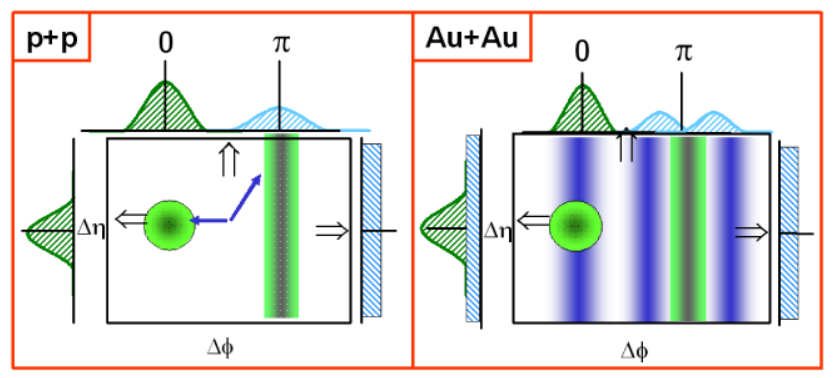

Fig. 1.1 A schematic illustration of the jet-induced di-hadron correlation signal in $\Delta \phi$ and $\Delta \eta$ and associated projections in $\Delta \phi$ or $\Delta \eta$ for (left) $p+p$ and (right) central $\mathrm{Au}+\mathrm{Au}$ collisions. The medium response components in $\mathrm{Au}+\mathrm{Au}$ is illustrated by fuzzy blue band

The modeling of energy transport to medium response is done in an ad hoc fashion, which necessary introduce a scale dependence. The ADS/CFT based approaches can describe both jet energy loss and Mach-cone like medium response in a single framework [9-11]. However it is not clear that these results can be extrapolated to QCD. The next improvement is to combine the calculation of jet quenching and medium response with the 3-D hydrodynamics framework [12].

How can we as experimentalist help with current situation? Given the vast amount of data and the experimental difficulties mentioned earlier, we feel it is imperative to reexam all the data and construct a global and self-consistent physical picture. A convincing picture should be able to connect results for high and low $p_{T}$, near- and away-side, and encompass different particle species and collision energies.

In the process of understanding underlying mechanisms for modification of jet correlation, we should not separate them from the mechanisms that are important for single particle spectra and flow measurements, such as hydrodynamic expansion and recombination. In fact, these bulk mechanisms should play a very important role in shaping the jet correlation pattern. The physical picture implied by correlation and bulk measurements should be consistent with each other. The second task of this manuscript is to exam the origin of triggers and the sources of correlated hadron pairs, such that we understand how the bulk production mechanism and medium response influence the interpretation of the correlation data.

In the end of the manuscript, we also discuss several experimental issues which are important for proper interpretations of the low $p_{T}$ correlation data.

\section{Correlation landscape}

The primary goal of the correlation analysis is to understand the interactions between jet and medium, i.e. jet quenching and the medium response. We now know that the jet quenching is responsible for the large suppression of the jet pairs at high $p_{T}$. The medium response is responsible for the enhancement of hadron pairs at low $p_{T}$. However most measurements typically focus on a particular momentum range or particular $\Delta \phi$ range. The methods and systematical errors associated with these measurements are not always the same, which makes the comparison of these results quite difficult. In a recent paper [6], PHENIX collaboration publish a detailed survey of the di-hadron correlation in broad ranges of trigger and partner $p_{T}$, which allows a systematic comparison of large amount data in a single analysis. Figure 2.1 shows the summary of the correlation landscape in $p_{T}^{A}, p_{T}^{B}$ and $\Delta \phi$. Many features can be identified at both the nearand away-side. They reflect in detail how the jet quenching and medium response vary with momentum and $\Delta \phi$.

A systematic approach to summarize these features can be done by dividing the $\Delta \phi$ distributions into three regions, an away-side head region $(|\Delta \phi-\pi|<\pi / 6)$, an away-side shoulder region $(\pi / 6<|\Delta \phi-\pi|<\pi / 3)$, and a near-side region $(\Delta \phi<\pi / 3)$, and then project them in the $\Delta \eta$ direction. The division of the away-side into the head and the shoulder regions facilitates the separation of the jet fragmentation contribution and medium response (the cone) at the away-side. The projection of near-side pairs in $\Delta \eta$ help us to separate the jet fragmentation contribution and medium response (the ridge) at the near-side. The $\Delta \eta$ projections for near-side pairs are shown in Fig. 2.2 for several representative $p_{T}^{A} \otimes p_{T}^{B}$ bins.

The information in Figs. 2.1 and 2.2 can be captured by a set of shape and yield variables. Jet shape variables include the ratio of head yield to shoulder yield $R_{\mathrm{H} S}$, Mach cone location $D$ or near-side width [2]. Jet yield variables include per-trigger yield (PTY) and $I_{A A}$ in the three $\Delta \phi$ ranges. A careful analysis of these variables as functions of $p_{T}^{A}$ and $p_{T}^{B}$ shows that all the features in Figs. 2.1 and 2.2 can be explained by the combined $p_{T}$ dependence of the jet fragmentation and medium response components at both the near- and away-side. The jet fragmentation contribution dominates at high $p_{T}$ and the medium response dominates at low $p_{T}$. The transitional region where the two sources roughly equal to each other can be approximated by $p_{T}^{A}+p_{T}^{B} \sim 6-8 \mathrm{GeV} / c$.

At the high $p_{T}$ region where the jet fragmentation dominates, we normally use the $I_{A A}$ to quantify the away-side suppression. It was observed that high $p_{T} I_{A A}$ is similar to single particle suppression, $R_{A A}[1,6]$. This observation is surprising at first sight, given that the away-side jets suffer on average more energy loss than that for inclusive jets. However, we should realize that the suppression level depends not only on the energy loss, but also on the jet spectral shape. As argued in [13], the away-side hadron spectra associated with a high $p_{T}$ trigger is much flatter than that of the inclusive hadrons. A flatter spectrum needs more energy loss to achieve a given level of suppression. Even though 


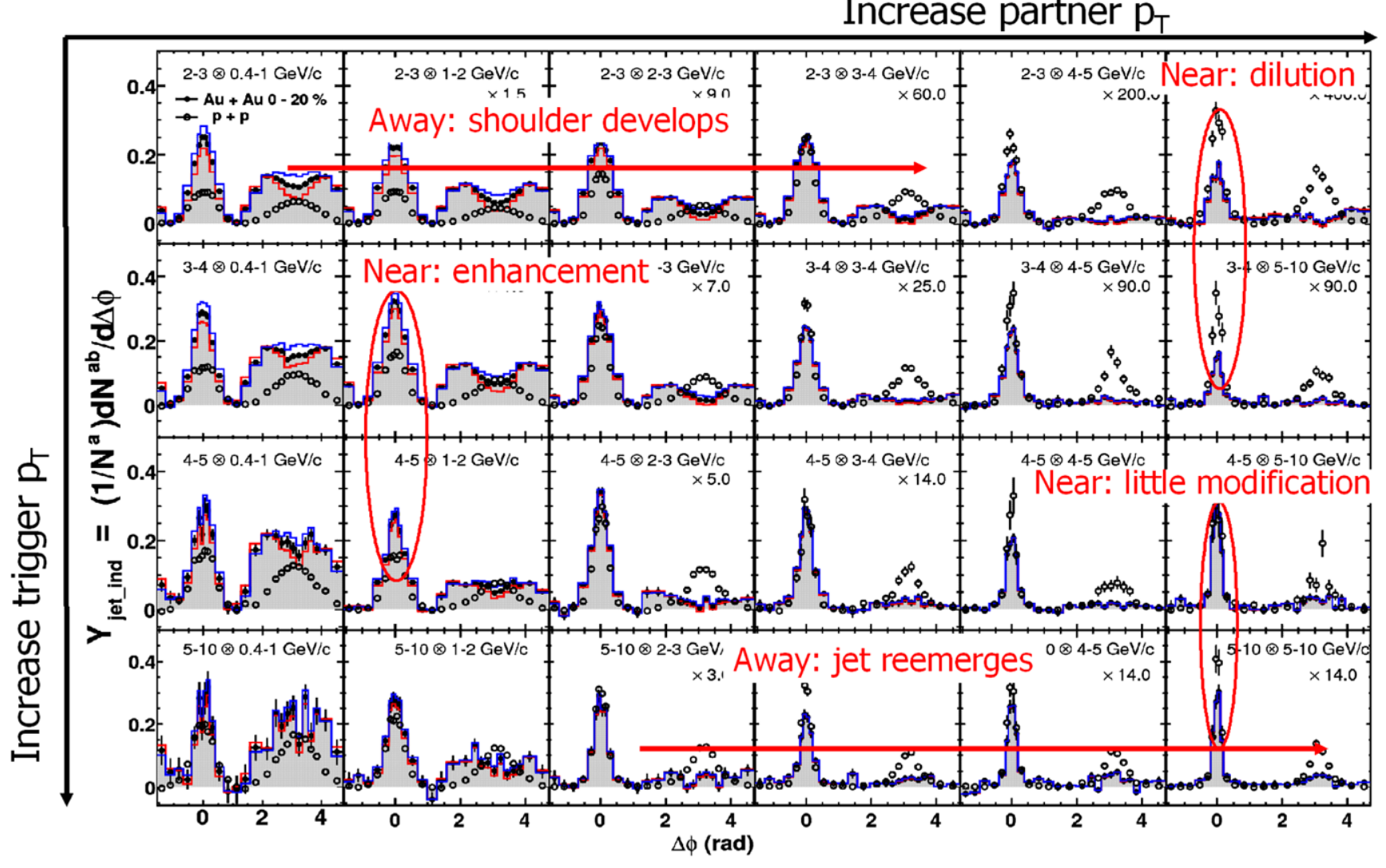

Fig. 2.1 The $\Delta \phi$ distribution in fine bin of trigger and partner $p_{T}$ in $p+p$ (open symbols) $0-20 \%$ central Au $+\mathrm{Au}$ (solid symbols) collisions, and thin lines indicate the systematic uncertainties. Several important features are indicated by the thick red lines and red circles

Fig. 2.2 Per-trigger yield versus $\Delta \eta$ for $p+p$ (open symbols) and $0-20 \%$ central $\mathrm{Au}+\mathrm{Au}($ filled symbols) collisions. Results are shown for four $p_{T}^{A} \otimes p_{T}^{\mathrm{B}}$ selections as indicated

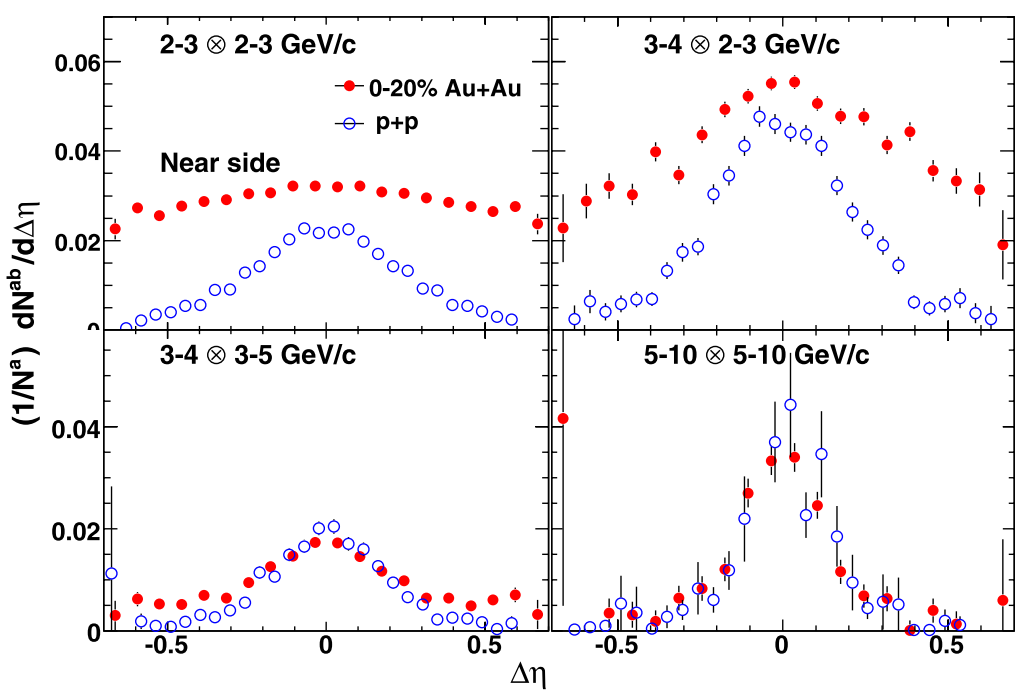

$I_{A A} \sim R_{A A}$, the away-side jets actually suffer on average $50 \%$ more energy loss than that for inclusive jets.

At the low $p_{T}$ region, medium response contributions, i.e. the cone on the away-side and the ridge on the near-side, play an important role in shaping the $\Delta \phi$ and $\Delta \eta$ distribution. A careful comparison between the ridge and the cone, after suppressing the jet fragmentation contribution, indicates that their properties are very similar. Both have similar spectral slopes, which are harder than inclusive hadrons but are softer than fragmentation hadrons [14]. Both have similar particle composition in terms of baryon to meson ratios $[15,16]$ : the ratios are bigger than that expected for 
Fig. 3.1 (Left) Inclusive charged hadron spectra from $p+p$ collisions compared with pQCD calculation down to $1.5 \mathrm{GeV} / c$ [18]. (Right) Low $p_{T}$ di-hadron correlation signal for charged hadrons in $p+p$. Both are for $\sqrt{s}=200 \mathrm{GeV}$

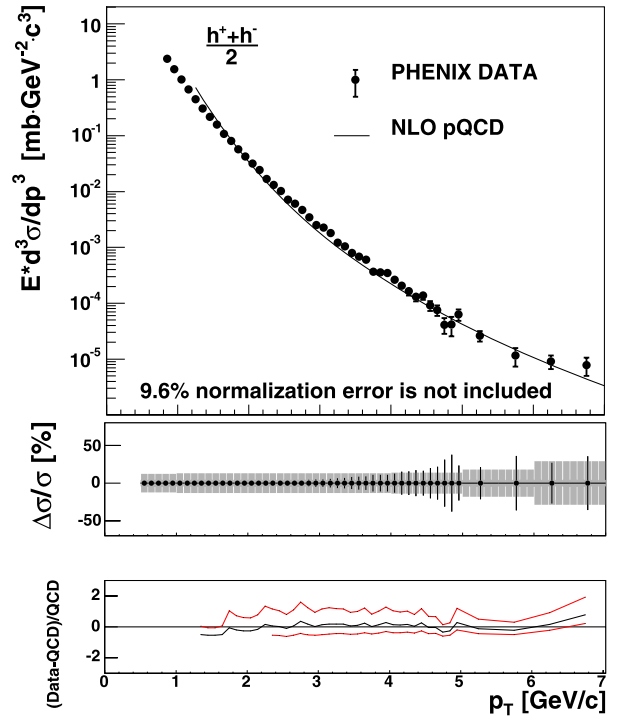

fragmentation and are close to that for the inclusive spectra (with large uncertainty). These similarities suggest that mechanisms for the ridge and the cone could be related. They also suggests that the bulk physics, hydrodynamical flow and parton recombination, should play an important role in this $p_{T}$ region.

Most previous low $p_{T}$ correlation analyses choose to use $I_{A A}$, the ratio of per-trigger yield in $\mathrm{Au}+\mathrm{Au}$ collisions to that in $p+p$ collision, to characterize the medium response. The rationale being that since each trigger tags one jet, the per-trigger yield is a good approximation to the per-jet yield (similar to high $p_{T}$ correlation analysis), thus $I_{A A}$ measures the modification of a jet in $\mathrm{Au}+\mathrm{Au}$ relative to that in $p+p$. As we shall show in the next section this assumption is not true at $p_{T}<5 \mathrm{GeV} / c$, and an alternative observable is needed for describing the medium response.

\section{Origin of triggers: jet fragmentation? Bulk? Or medium response?}

In $p+p$ collisions at RHIC energies, data from the single particle spectra and di-hadron correlation suggest that most hadrons above $2 \mathrm{GeV} / c$ originate from jet fragmentation. This is attested by the fact the particle spectra are well described by the pQCD calculation $[17,18]$ and the correlation function show strong jet-like signals down to very low $p_{T}[6,19]$ as shown by Fig. 3.1.

The situation is very different in heavy ion collisions. At $p_{T}<5 \mathrm{GeV} / c$ where medium response is important, the inclusive hadron yield is dominated by non-perturbative bulk production mechanisms such as hydrodynamic flow and recombination. The experimental evidences include: a particle composition that is strongly modified relative to that in $p+p$ collisions [20], a $R_{A A}$ that peaks around $p_{T} \sim 3 \mathrm{GeV} / c$ at a level much bigger than what is suggested by jet quenching [21], and a hadron elliptic flow that reaches maximum in this momentum region at a level much bigger than what one expect from the jet quenching [22]. These results imply that the origins of the hadrons at $p_{T}<5 \mathrm{GeV} / c$ in $\mathrm{Au}+\mathrm{Au}$ collisions are very different from those in $p+p$. Thus per-trigger yield and $I_{A A}$ are not good observables for the medium response since the triggers are also modified. According to hydro + coalescence model, a large fraction of the triggers at $p_{T}<5 \mathrm{GeV} / c$ may come from thermalthermal or thermal-shower recombination. The former has no jet correlation, while the latter can retain some jet correlation which can be modified by the flow [23].

If most hadrons do not originated from jet fragmentation, and have either no correlation or reduced correlation strength, they should lead to a dilution of per-trigger yield in $\mathrm{Au}+\mathrm{Au}$ collisions relative to $p+p$. In fact PHENIX data indicate the effect of the dilution on the per-trigger yield [24]. It is best illustrated with the near-side $\Delta \eta$ correlation as shown in Fig. 3.2. ${ }^{1}$ We estimate dilution factor $(\sim 2)$ for $3-4 \mathrm{GeV} / c$ triggers based on their correlations with $5-10 \mathrm{GeV} / c$ hadrons as shown by the inserted panel: requiring $5-10 \mathrm{GeV} / c$ hadrons ensures the pairs are dominated by the jet fragmentation (left panel of the insert), thus deviation of $I_{A A}$ from one for soft triggers reflects the level of dilution (the red arrow). Once the dilution factor is corrected, we subtract out the jet fragmentation contribution and obtain the ridge distribution (black filled circles). The estimated ridge contribution is approximately flat, consistent with experimental data at large $\Delta \eta$ [5]. However, this dilution effect was not observed in some STAR analyses [5, 25],

\footnotetext{
${ }^{1}$ The data are from Fig. 2.2.
} 
Fig. 3.2 Per-trigger yield $\Delta \eta$ distribution for 3-4 GeV/c triggers and two parter $p_{T}$ selections. The ridge distribution (solid circles) is estimated by subtracting the $\mathrm{Au}+\mathrm{Au}$ distribution corrected by dilution effect (open squares) minus the $p+p$ (open circles). The dilution correction $(\times 2)$ is indicated by the red arrow in the insert (see text for explanation)

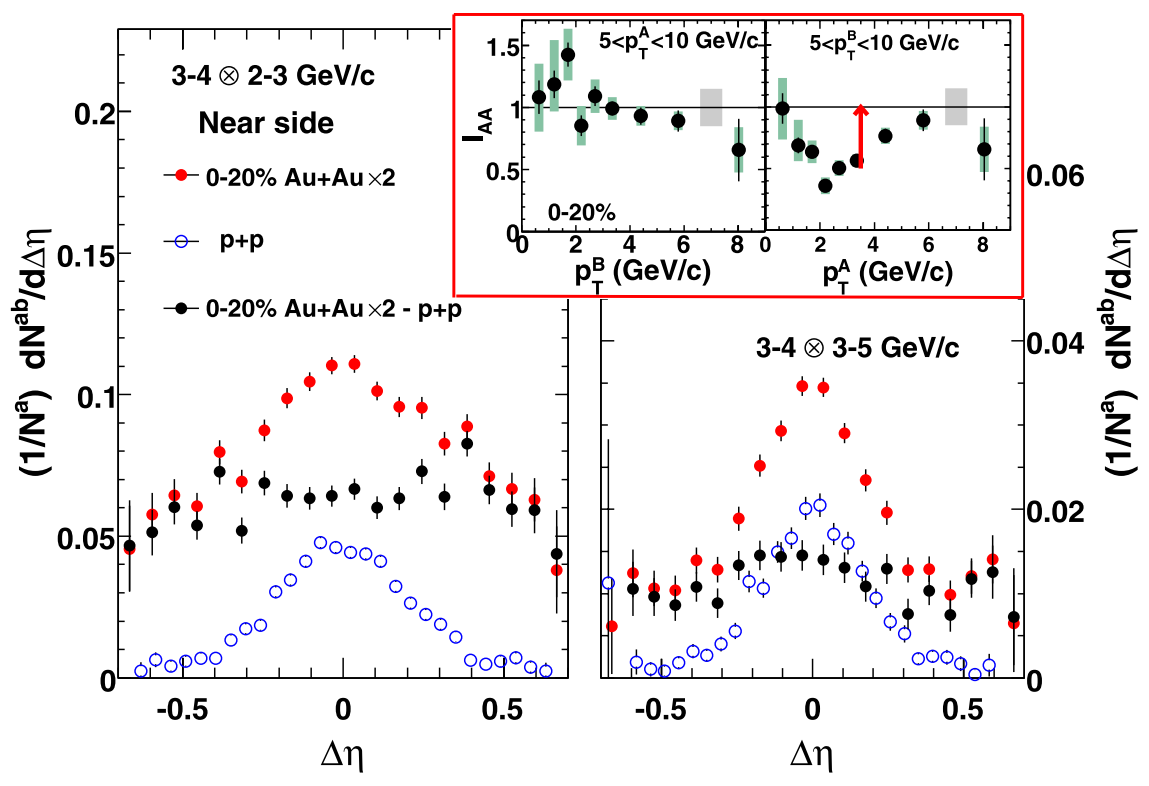

which showed that the $\mathrm{PTY}_{A A}$ subtracted by the estimated ridge equals $\mathrm{PTY}_{p p}$ before any correction for dilution effect.

Many previous analyses assume that most triggers originated from jet fragmentation. This naturally leads to different interpretations for the medium responses between the near- and away-side. The near-side pairs were thought to be surface biased and should have smaller modifications, while the away-side pairs traverse on average a longer path, thus should be strongly modified. However, this interpretation is not correct if most triggers come from the bulk or medium response. In this case, the triggers are not surfaced biased but are emitted from the whole volume of the overlap region. We shall elaborate on this point in the next section.

The interpretation of the PTY as a function of angle with respect to the reaction plane, $\phi-\Phi_{R P}$, should also be modified if non-jet triggers dominate. This is because the variation of trigger yield with $\phi-\Phi_{R P}$ (characterized by $v_{2}$ ) is much larger than what is expected from fragmentation of survived jets. Thus the dilution of PTY could also depend on $\phi-\Phi_{R P}$.

A physical observable better than PTY for describing the medium response is the hadron pair yield JPY, the total number of correlated pairs per-event, introduced in [6]. The modification of pair yield in $\mathrm{Au}+\mathrm{Au}$ collision can be characterized by $J_{A A}$ :

$$
\begin{aligned}
& J_{\mathrm{AA}}\left(p_{\mathrm{T}}^{\mathrm{a}}, p_{\mathrm{T}}^{\mathrm{b}}, \Delta \phi\right) \\
& =\frac{\mathrm{JPY}}{\left\langle N_{\text {coll }}\right\rangle \mathrm{JPY} \mathrm{JPY}^{p+p}} \\
& =\frac{1}{\sigma_{\mathrm{A}+A}} \frac{d^{3} \sigma_{\mathrm{j} e t \_i n d}^{\mathrm{A}+\mathrm{A}}}{d p_{\mathrm{T}}^{\mathrm{a}} d p_{\mathrm{T}}^{\mathrm{b}} d \Delta \phi} / \frac{\left\langle N_{\text {coll }}\right\rangle}{\sigma_{p+p}} \frac{d^{3} \sigma_{\text {jet_ind }}^{p+p}}{d p_{\mathrm{T}}^{\mathrm{a}} d p_{\mathrm{T}}^{\mathrm{b}} d \Delta \phi}
\end{aligned}
$$
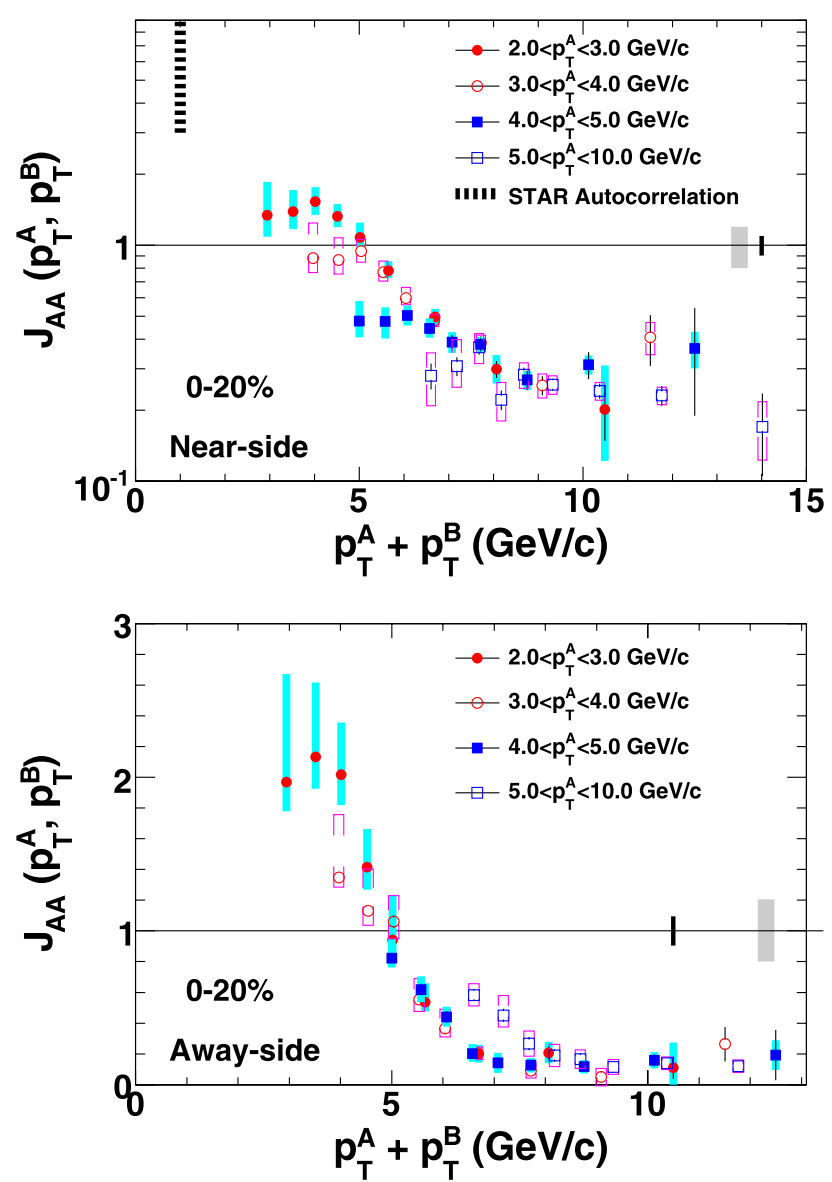

Fig. 3.3 The modification factor for hadron pair yield as function of $p_{T}^{\text {sum }}=p_{T}^{A}+p_{T}^{B}$ for the near-side (top) and away-side (bottom). $p_{T}^{\text {sum }}$ condenses the 2-D correlation data in $p_{T}^{A}$ and $p_{T}^{B}$ space into a one dimensional plot. The STAR auto-correlation result [26] is divided by 3 (the lower end) to normalize the $\eta$ acceptance relative to PHENIX 
$J_{A A}$ quantify the medium modification of hadron pair yield from the expected yield, in a way similar to $R_{A A}$ for describing the modification of single hadron yield. The hadron pair yield is proportional to the di-jet yield, and in the absence of nuclear effects, it should scale with $N_{\text {coll }}$, and $J_{A A}=1$. Figure 3.3 shows $J_{A A}$ as a function of pair proxy energy $\left(p_{T}^{\text {sum }}=p_{T}^{A}+p_{T}^{B}\right)$ for the near- (top panel) and away-side (bottom panel). In contrast to a constant suppression at large $p_{T}^{\text {sum }}$, the pair yields are less suppressed at $p_{T}^{\text {sum }}<6-8 \mathrm{GeV} / c$. This reflects directly the energy transport that redistributes energy of the quenched jets to low $p_{T}$ hadrons (i.e. medium response). We would like to point out that $p_{T}^{\text {sum }}$ is a natural variable for the near-side correlation since it approximates the original jet energy. In fact the data show an approximate scaling in $p_{T}^{\text {sum }}$. Even the away-side data tend to group together, because the medium response increases with away-side jet energy which in turn increases with both $p_{T}^{A}$ and $p_{T}^{B}$.

\section{Consequence of triggering on medium response}

As argued above, the soft triggers (at $p_{T}<5 \mathrm{GeV} / c$ ) have three possible origins: jet fragmentation, hadrons that have no correlation which leads to dilution of per-trigger yield, or triggers come directly from medium response (e.g. the cone and the ridge). In many theoretical models, the energy loss processes lead to heating of the partons in the local medium. This local heating effectively boosts bulk partons to higher energy, which then hadronize into particles that are correlated with the quenched jets. Since this type of mechanisms does not require new particle production, they are more effective in generating large yield of correlated pairs than those involving gluon radiation. However, this contribution has a steeper slope in $p_{T}$ than the jet fragmentation contribution. Thus it is important at $p_{T}<5 \mathrm{GeV} / c$, but become less important at higher $p_{T}$. In a simple jet absorption picture, the yield of fragmentation hadrons from survived jets scales with $R_{A A, o}$ (the constant suppression factor at high $p_{T}$ ). The yield of medium response hadrons should scale with the number of quenched jets, i.e. $1-R_{A A, o}$.

In general, hadrons in correlated pairs can be chosen from either survived jets or quenched jets. Therefore, jetinduced pairs can be divided into three groups. Jet-jet pair: both hadrons come from fragmentation of survived jets. Jetmedium pair: one hadron comes from fragmentation of survived jet, the second hadron comes from medium feedback of quenched jet. Medium-medium pair: both hadrons come from medium feedback of quenched jets. The rates of their contributions to inter-jet pairs scale approximately as $R_{A A, o}^{2}, R_{A A, o}\left(1-R_{A A, o}\right)$ and $\left(1-R_{A A, o}\right)^{2}$. Clearly, in this simple picture, the stronger the suppression is, the larger the contribution from medium-medium pairs is. In the limit $R_{A A, o} \rightarrow 0$, the medium-medium contribution naturally dominates.

We performed a simple simulation based on jet absorption picture [27] to investigate contributions from the three sources, focusing on the medium-medium pairs which were not considered in many previous models. The details of the simulation can be found in [28]. The idea is to generate dijets according to collision density distribution in the overlap plane $\left(\rho_{\text {ncoll }}(x, y)\right)$, and attenuate them as they traverse a medium whose density is proportional to the participant nucleon density $\left(\rho_{\text {npart }}(x, y)\right)$ [27]. Both $\rho_{\text {ncoll }}$ and $\rho_{\text {part }}$ were generated via a Monte-Carlo Glauber model code. If a jet survives the medium, it is converted into $N_{\text {jet }}$ hadron via fragmentation, otherwise it is converted into $N_{\text {med }}$ medium response hadrons. $N_{\text {jet }}$ and $N_{\text {med }}$ are assumed to have a Poisson distribution with a mean of 1 and 2 respectively. The former corresponds to multiplicity of hadron in $1-4 \mathrm{GeV} / c$ from a $6 \mathrm{GeV} / c$ jet. The latter is chosen to take into account the observed enhancement in $1-4 \mathrm{GeV} / c$ seen in the data. Figure 4.1 compares the yields for jet-jet, jetmedium and medium-medium pairs as a function of centrality. The medium-medium contribution increases with $N_{\text {part }}$ and dominates the yield in central collisions.

To describe the $\Delta \phi$ distribution, we adjust the jet fragmentation kinematics to match the $p+p$ correlation data [19]. We also assume that only $30 \%$ of the inter-jet pairs are detected, so as to account for finite detector acceptance and the swing of the away-side jet in pseudo-rapidity. The medium response hadrons are assumed to be emitted equally at $\pi \pm D(D=1.1)$. This step makes sure that the shape of jet-jet and jet-medium pairs, those included in traditional models, matches the measured away-side distribution, so we can study the impact of medium-medium pairs to the $\Delta \phi$ distribution.

The emission directions for hadrons contributing to medium-medium pairs are illustrated by left panel of Fig. 4.2. In this picture, both jets are quenched and converted into

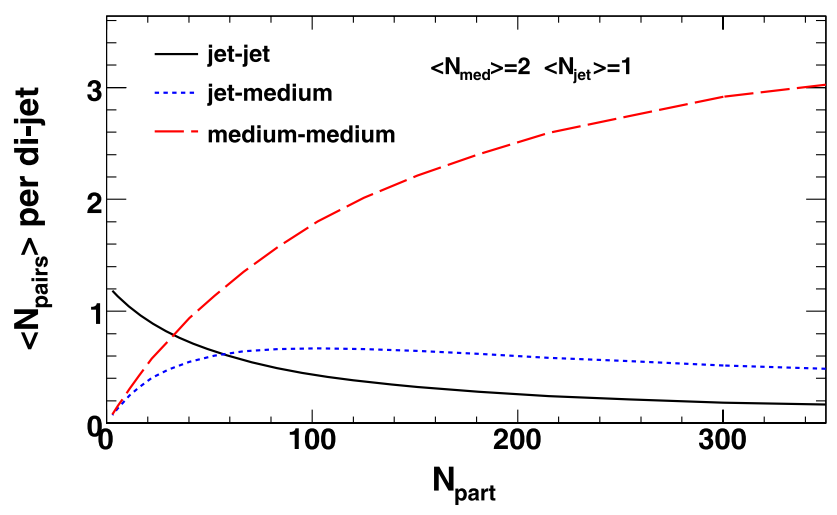

Fig. 4.1 The centrality dependence of pair yield normalized by number of dijets from jet-jet, jet-medium and medium-medium sources 
Fig. 4.2 $\Delta \phi$ distributions in $0-5 \%$ central $\mathrm{Au}+\mathrm{Au}$ collisions for medium-medium pairs. The diagram at the left illustrates the typical emission directions (solid arrows) and $\Delta \phi$ values (numbers)
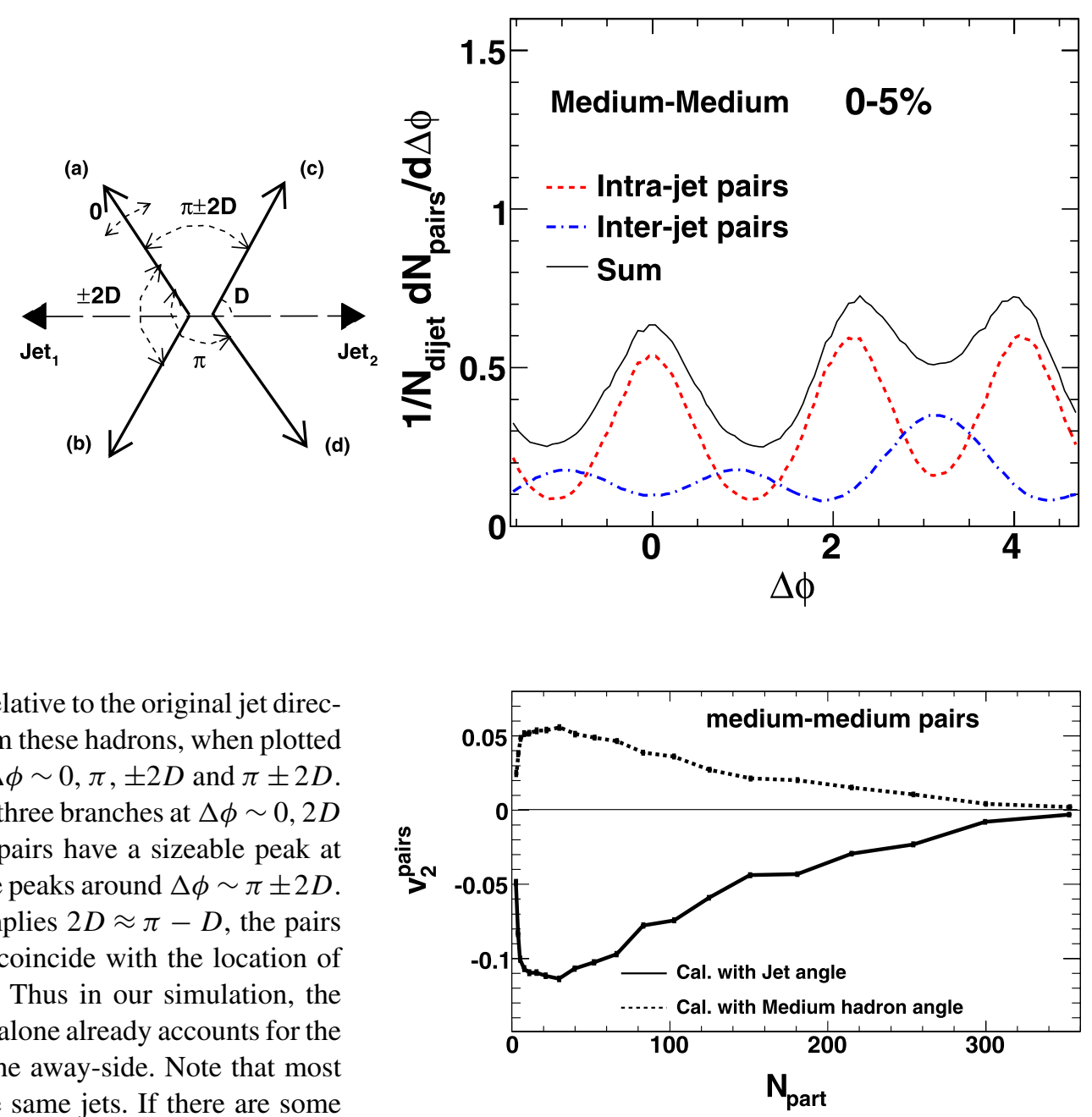

Fig. 4.3 The $v_{2}$ for and medium-medium pairs calculated as $\left\langle\cos 2\left(\phi-\Psi_{R P}\right)\right\rangle$, where $\phi$ is the azimuthal angle of jets (solid lines) or medium response hadrons (dotted lines)

rameter, should be useful in helping us to constrain the underlying mechanisms of the medium response.

We also studied the consequence of triggering on medium response for three particle correlation. There are three types of triplets: jet-jet-jet $(\mathrm{j}-\mathrm{j}-\mathrm{j})$ triplets, jet-medium-medium (j-m-m), jet-jet-medium (j-j-m) triplets and mediummedium-medium $(\mathrm{m}-\mathrm{m}-\mathrm{m})$ triplets. The standard coordinate system $[29,30]$ is adopted, i.e, we plot the azimuth angle difference between particle 1 and $2\left(\Delta \phi_{12}\right)$ vs azimuth angle difference between particle 1 and $3\left(\Delta \phi_{13}\right)$ as shown in Fig. 4.4. The $\mathrm{j}-\mathrm{j}-\mathrm{m}$ and $\mathrm{j}-\mathrm{m}-\mathrm{m}$ contributions lead to the conventional off-diagonal terms which were used to identify the Mach-cone pattern in the data [30,31]. The dominating contribution comes from m-m-m term, which has a more complicated shape due to the large deflection angle w.r.t the original jet direction. The $\mathrm{m}-\mathrm{m}-\mathrm{m}$ term appears as several broad peaks in the $\Delta \phi_{1,2}$ and $\Delta \phi_{1,3}$ plane. It's away-side peak is so spread out that it shadows the modstead of the per-trigger yield), and the corresponding $v_{2}$ pa- 
Fig. 4.4 Azimuthal distributions for triplets from various sources in $0-5 \%$ $\mathrm{Au}+\mathrm{Au}$ collisions

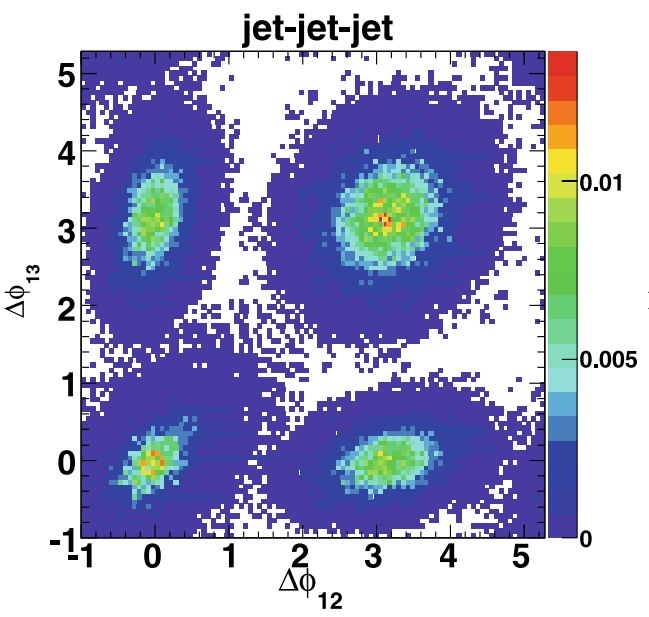

jet-medium-medium, jet-jet-medium

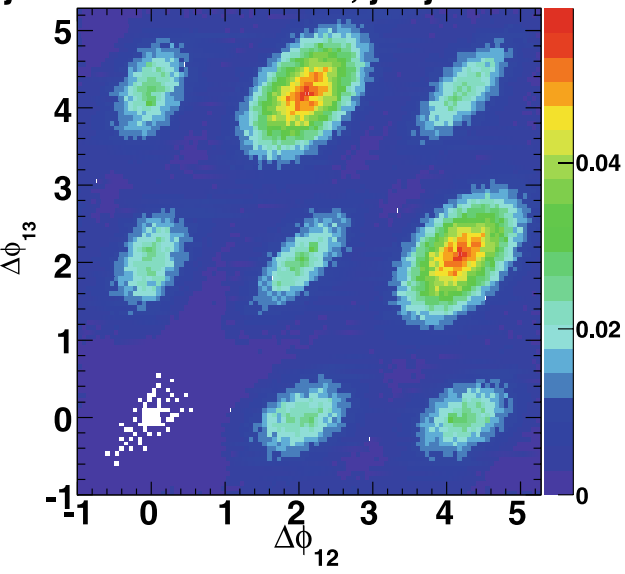

medium-medium-medium
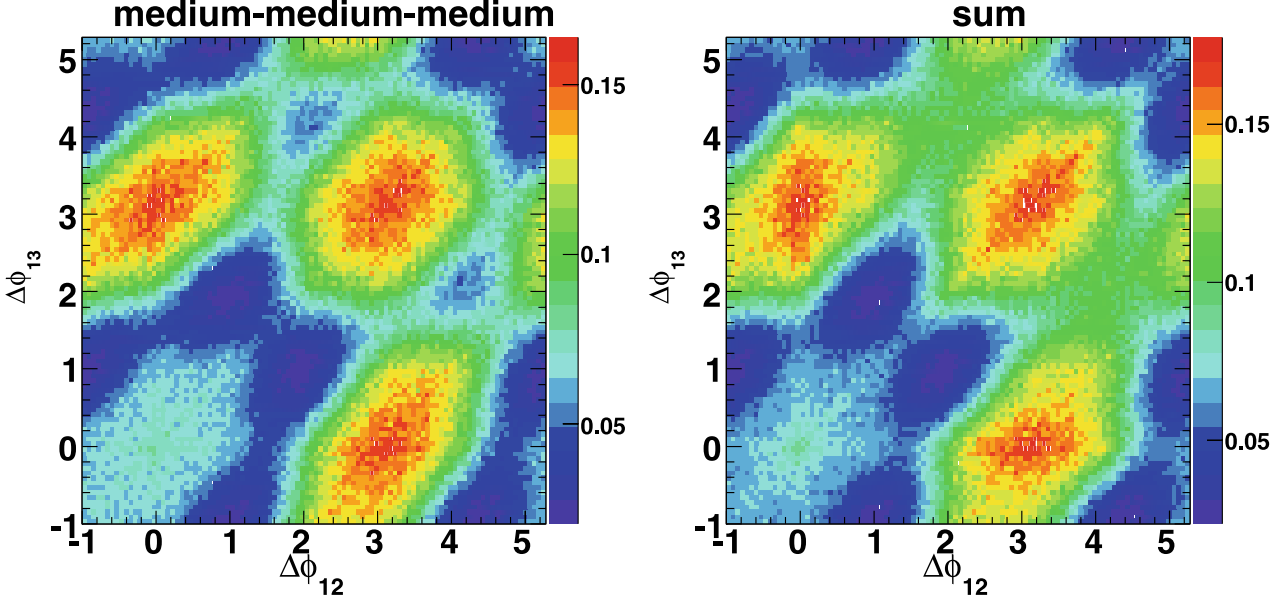

est off-diagonal contribution from $\mathrm{j}-\mathrm{m}-\mathrm{m}$ and $\mathrm{j}-\mathrm{j}-\mathrm{m}$ terms. Clearly, the shape of the three-particle correlation signal depends strongly on whether we trigger on jet fragmentation (j-m-m, j-j-m) or medium response (m-m-m), even though the correlation shape from medium-medium and jet-medium can be similar. Our study shows that the interpretation of the three particle correlation results are complicated if the triggers come from the medium response.

\section{Additional thoughts}

Most di-hadron correlation analyses rely on the following two-source model formula to obtain the jet signal by subtracting the background term $[4,6]$ :

$$
C(\Delta \phi)=J(\Delta \phi)+\xi\left(1+2 v_{2}^{A} v_{2}^{B} \cos 2 \Delta \phi\right)
$$

where $\xi$ is the background level, which is a number close to one in central collisions. This approach implicitly assumes that either there is no jet-flow cross-term, or the crossterm is included in the definition of the jet function $J(\Delta \phi)$. Currently, the main debate over (5.1) centers on what $v_{2}$ values to use for triggers $\left(v_{2}^{A}\right)$ and partners $\left(v_{2}^{B}\right)$. This debate is caused by a proliferation of different flow measurements, designed to remove non-flow bias and/or event-byevent fluctuation effects. In our opinion, these additional sources contribute to $C(\Delta \phi)$, but they should not be included in the jet function $J(\Delta \phi)$. Instead, they should be included in the flow background, i.e. the $v_{2}^{A}, v_{2}^{B}$ used in the background subtraction should include various non-flow effects (other than jet) and event by event fluctuations. If we do not include them in the flow background, then we would need to subtract their contributions later from the jet yield. ${ }^{2}$

The $v_{2}$ values used in the background subtraction are different between STAR and PHENIX. PHENIX collaboration uses reaction plane $v_{2}$ based on forward detectors. STAR collaboration use the average of reaction plane $v_{2}$ and four particle culminant $v_{2}$ [3]. The latter is about $20 \%$ smaller than the former, and the difference between the two is quote as the systematic error. As a result, the $v_{2}$ used by STAR is about $10 \%$ smaller than that for PHENIX, but their dif-

\footnotetext{
${ }^{2}$ The only caveat is that the non-flow and event by event contributions are assumed to have $\cos 2 \Delta \phi$ shape. Based on how the $C(\Delta \phi)$ varies with angle w.r.t reaction plane [32], it is a fairly good assumption.
} 
Fig. 5.1 Correlation for 4-6 $\otimes 0.15-4 \mathrm{GeV} / c$ from STAR collaboration in $d+\mathrm{Au}$ and $0-5 \% \mathrm{Au}+\mathrm{Au}$ collisions [33]: (a) the per-trigger yield distribution in $\Delta \phi,(\mathbf{b})$ the mean $p_{T}$ of partners as function of $\Delta \phi$

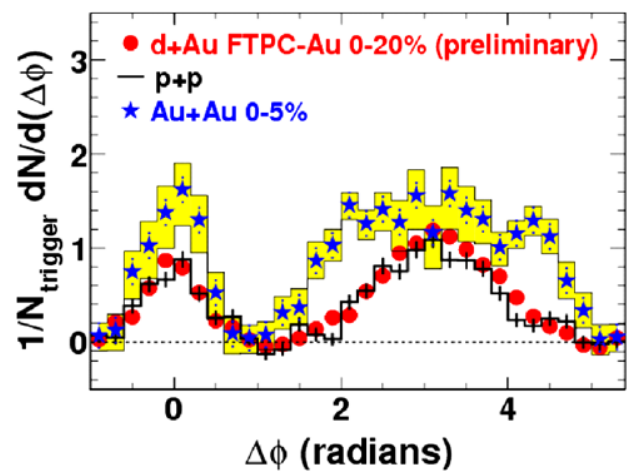

(a)

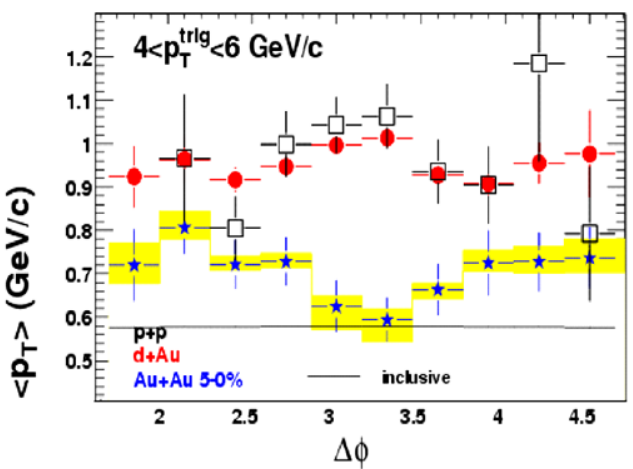

(b)

Fig. 5.2 The $\Delta \phi$ distribution in central collisions for three collision energies in central collisions

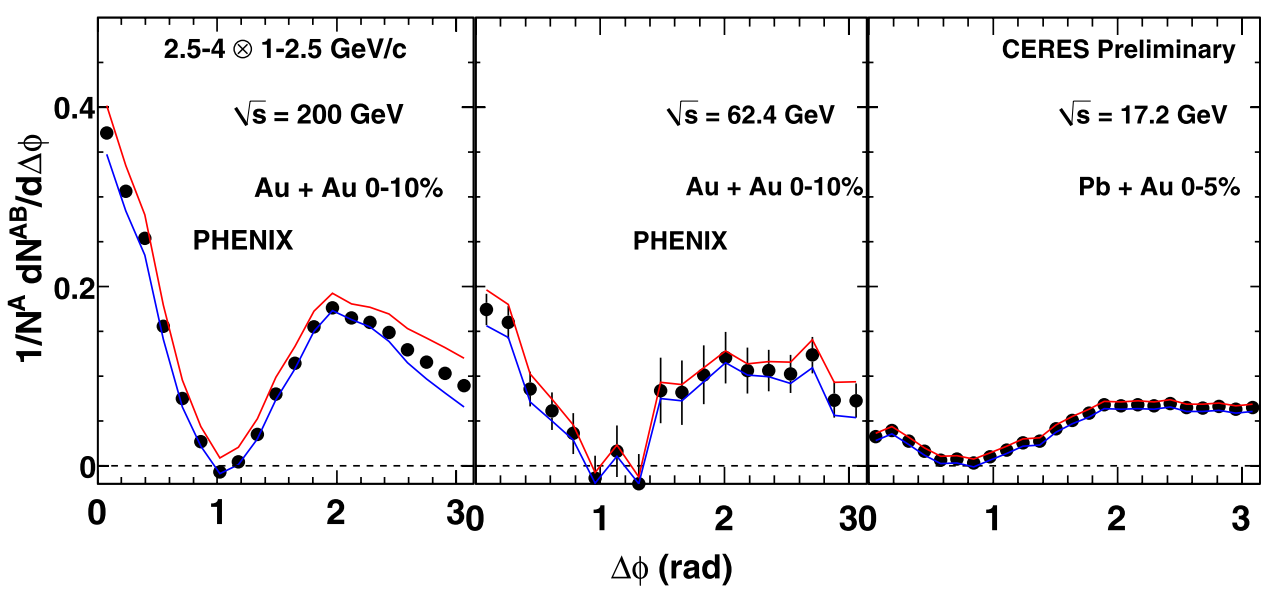

ferences is covered by the large $v_{2}$ error quoted by STAR. But because of this difference, STAR collaboration does not see a very pronounced dip in the $d N / \Delta \phi$ distribution as in PHENIX (Fig. 5.1a). However, they show that if one chose a different quantity, such as the mean $p_{T}$ which characterizes the energy flow, one observes a clear dip at $\Delta \phi \sim \pi$ (Fig. 5.1b). This shows that away-side shape also depends on the physical observable. Existence of a dip in multiplicity distribution is not a sufficient condition for Mach cone, because it could be filled up by the punch-through jet, or the jet wake.

Important insights on the origin of the cone and the ridge have been provided by a energy scan from SPS to RHIC. A strong modification of the away-side correlation was observed at the top SPS energy $\left(\sqrt{s_{N N}}=17.2 \mathrm{GeV}\right)$ [34]. There, the strong away-side broadening has been used to argue for a similar interpretation (such as Mach cone) as for results at RHIC. However a quantitative analysis of the energy dependence of the modification patterns (see Fig. 5.2) shows that the yield of medium response are quite different between RHIC and SPS energies. The near-side yield drop by almost factor of 8 going from 200 to $17 \mathrm{GeV}$, the away-side shoulder yield drops by a factor of 2 in the same energy range, while little dependence of the yield on $\sqrt{s}$ is observed for the away-side head region, where the jet fragmentation is expected to be important. This suggests a much weaker medium response at SPS energy (the ridge almost disappeared and cone strongly suppressed) than that at RHIC, which probably implies different mechanisms are in play at SPS energy, such as a stronger Cronin effect combined with a weaker energy loss at lower energy [35].

\section{Conclusion}

First several years of RHIC data on jet-induced particle correlations have revealed a fascinating and rich set of insights on the mechanisms for jet medium interactions. The $p_{T}$, PID and centrality dependence of the correlation patterns are observed to be consistent with competition between jet fragmentation and medium response. Fragmentation of survived jets dominates the correlation signal at high $p_{T}$, while the medium response to quenched jets dominates the correlation signal at low $p_{T}$. It is also observed that the medium response at the near-side (the ridge) and the away-side (the cone) share similar properties. 
Understanding the medium response requires knowledge of the origins of the hadrons in the pairs at $p_{T}<5 \mathrm{GeV} / c$. Both the spectra and correlation results suggest we may be triggering on medium response at intermediate $p_{T}$. A simple jet absorption model is employed to investigate the roles of pairs from medium response (medium-medium pair) on the pair shape and pair yield. The correlations among medium response particles were shown to dominate the pair yield at intermediate $p_{T}$, while preserving the di-hadron correlation patterns in $\Delta \phi$. These medium-medium pairs are expected to have significantly reduced anisotropy due to the smearing caused by the large emission angle w.r.t the original jet direction. The model suggests a common mechanistic origin for the ridge and the cone, and provides a natural explanation for their similarities.

We also touched on the flow background subtraction in the two-source model framework. We argue that the nonflow effects and event by event fluctuation effects should not be taken out of the $v_{2}$ used in the subtraction. We also show that the shape of the away-side $\Delta \phi$ distribution shows a more significant dip in mean $p_{T}$ than in multiplicity. Finally the energy scan data suggests that the medium response mechanisms might be different at SPS from those at RHIC energies.

\section{References}

1. J. Adams et al. (STAR Collaboration), Phys. Rev. Lett. 97, 162301 (2006)

2. A. Adare et al. (PHENIX Collaboration), Phys. Rev. C 77, 011901(R) (2008)

3. J. Adams et al. (STAR Collaboration), Phys. Rev. Lett. 95, 152301 (2005)

4. S.S. Adler et al. (PHENIX Collaboration), Phys. Rev. Lett. 97, 052301 (2006)

5. J. Putschke, J. Phys. G 34, S679 (2007), see Fig. 5

6. A. Adare et al. (PHENIX Collaboration), Phys. Rev. C 78, 014901 (2008)
7. J. Casalderrey-Solana, E.V. Shuryak, D. Teaney, hep-ph/0602183 (2006)

8. T. Renk, K.J. Eskola, Phys. Rev. C 75, 054910 (2007)

9. S.S. Gubser, D.R. Gulotta, S.S. Pufu, F.D. Rocha, J. High Energy Phys. 0810, 052 (2008)

10. P.M. Chesler, L.G. Yaffe, Phys. Rev. Lett. 99, 152001 (2007)

11. J.J. Friess, S.S. Gubser, G. Michalogiorgakis, S.S. Pufu, Phys. Rev. D 75, 106003 (2007)

12. https://wiki.bnl.gov/TECHQM/index.php/TECHQM:About

13. J. Jia (PHENIX Collaboration), Int. J. Mod. Phys. E 16, 2000 (2007)

14. A. Franz (PHENIX Collaboration), J. Phys. G 35, 104002 (2008)

15. S. Afanasiev et al. (PHENIX Collaboration), Phys. Rev. Lett. 101, $082301(2008)$

16. J. Bielcikova (STAR Collaboration), arXiv:0806.2261 [nucl-ex] (2008)

17. S.S. Adler et al. (PHENIX Collaboration), Phys. Rev. Lett. 91, 241803 (2003)

18. S.S. Adler et al. (PHENIX Collaboration), Phys. Rev. Lett. 95, 202001 (2005)

19. S.S. Adler et al., Phys. Rev. C 73, 054903 (2006)

20. S.S. Adler et al. (PHENIX Collaboration), Phys. Rev. C 69, 034909 (2004)

21. S.S. Adler et al. (PHENIX Collaboration), Phys. Rev. C 69, 034910 (2004)

22. J. Adams et al. (STAR Collaboration), Phys. Rev. Lett. 93, 252301 (2004)

23. R.J. Fries, S.A. Bass, B. Muller, Phys. Rev. Lett. 94, 122301 (2005)

24. J. Jia, J. Phys. G 35, 104033 (2008)

25. J. Bielcikova, J. Phys. G 34, S929 (2007)

26. M. Daugherity (STAR Collaboration), J. Phys. G 35, 104090 (2008)

27. A. Drees, H. Feng, J. Jia, Phys. Rev. C 71, 034909 (2005)

28. J. Jia, R. Lacey, Phys. Rev. C 79, 011901(R) (2009)

29. N.N. Ajitanand (PHENIX Collaboration), Acta Phys. Hung. A 27, 197 (2006)

30. B.I. Abelev et al. (STAR Collaboration), arXiv:0805.0622 [nuclex] (2008)

31. T. Renk, J. Ruppert, Phys. Rev. C 76, 014908 (2007)

32. J. Jia (PHENIX Collaboration), AIP Conf. Proc. 828, 219 (2006)

33. F. Wang, J. Phys. Conf. Ser. 27, 32 (2005)

34. M. Ploskon (CERES Collaboration), Nucl. Phys. A 783, 527 (2007)

35. A. Adare et al. (PHENIX Collaboration), Phys. Rev. Lett. 101, $162301(2008)$ 